\title{
Certain Simple Maximal Subfields in Division Rings
}

\author{
M. Aaghabali ${ }^{a}$, M.H. Bien ${ }^{\mathrm{b}}$ \\ a School of Mathematics, University of Edinburgh, Edinburgh EH9 3JZ, Scotland \\ ${ }^{\mathrm{b}}$ Faculty of Mathematics and Computer Science, University of Science, VNU-HCM, 227 Nguyen Van Cu Str., \\ Dist. 5, HCM-City, Vietnam
}

\begin{abstract}
Let $D$ be a division ring finite dimensional over its center $F$. The goal of this paper is to prove that for any positive integer $n$ there exists $a \in D^{(n)}$, the $n$-th multiplicative derived subgroup, such that $F(a)$ is a maximal subfield of $D$. We also show that a single depth- $n$ iterated additive commutator would generate a maximal subfield of $D$.
\end{abstract}

AMS Classification: 16K20, 16R50, 17A35.

Keywords: Division Ring, Rational Identity, Maximal Subfield.

\section{Preliminary}

Throughout this paper $D$ is a division ring with center $F$. An element $a \in D$ is called algebraic over $F$, if there exists a non-zero polynomial $a_{0}+a_{1} x+\cdots+a_{n} x^{n}$ over $F$ such that $a_{0}+a_{1} a+\cdots+a_{n} a^{n}=0$. If $a \in D$, then $F(a)$ denotes the subfield of $D$ generated by $F$ and $\{a\}$. For a (multiplicative) group $G$ we denote by $(a, b)=a b a^{-1} b^{-1}$ the multiplicative commutator of $a, b \in G$ and $(G, G)$ the multiplicative commutator subgroup of $G$. We denote by $G \supseteq G^{\prime} \supseteq \cdots \supseteq G^{(n)} \supseteq \ldots$ the derived series of $G$, that is, $G^{\prime}=(G, G)$, and $G^{(n+1)}=\left(G^{(n)}, G^{(n)}\right)$ for every $n \geq 1$. For a unital associative $\operatorname{ring} R$ we use $[a, b]=a b-b a$ to denote the additive commutator of $a, b \in R$ and $R_{1}=[R, R]$ the additive commutator subgroup of $R$. We denote by $R \supseteq R_{1} \supseteq \ldots \supseteq R_{n} \supseteq \ldots$ the additive derived series of $R$, that is, $R_{1}=[R, R]$, and $R_{n+1}=\left[R_{n}, R_{n}\right]$ for every $n \geq 1$. For a given division ring $D$ we call $D^{(n)}$ and $D_{n}$ the $n$-th multiplicative and additive derived groups of $D$, respectively. In the case of division ring $D$ we simply use $D^{\prime}$ and $[D, D]$ to denote the multiplicative and additive group of commutators in $D$, respectively. If $A$ is a subset of $D$ we use $A^{*}$ to denote $A \backslash\{0\}$. A subfield $K$ of $D$ is called a maximal subfield if $K$ is its own centralizer in $D^{*}$. We denote by $\operatorname{dim}_{F} D$ the dimension of $D$ over $F$. If $\operatorname{dim}_{F} D=n^{2}$, then $n$ is called the degree of division ring $D$. By $M_{n}(K), G L_{n}(K)$ and $S L_{n}(K)$ we mean all square matrices, all invertible matrices and all matrices of determinant one of order $n$ with entries from $K$, respectively.

Mahdavi-Hezavehi in [12] investigates the algebraic properties of the multiplicative group of commutators in a division ring and shows that any subfield $K$ of a division ring $D$ which is separable over the center of $D$ is generated over the center by a commutator subgroup of $D^{\prime}$. Afterwards, Mahdavi-Hezavehi and his colleagues in [13] studied other generating properties of commutator subgroup and showed that each finite separable extension of the center of $D$ could be considered as a simple extension $F(c)$, where $c$ is an element in $D^{\prime}$. Now, it is natural to consider similar questions in terms of some other elements coming from certain substructures of a division ring. In particular, one can pose the following questions:

Question 1. 11, Problems 28, 29] Let $D$ be a division ring finite dimensional over its center $F$.

(i) Whether for any non-central normal subgroup $N$ of $D^{*}$ does there exist element $c \in N$ such that $F(c)$ is a maximal subfield in $D$ ?

(ii) Whether for any non-central subnormal subgroup $N$ of $D^{*}$ does there exist element $c \in N$ such that $F(c)$ is a maximal subfield in $D$ ?

In this note we rely on rational identities to show that some maximal subfields are generated by elements coming from $D^{(n)}$ and $D_{n}$, resp. $n$-th derived subgroup of $D^{*}$ and $n$-th iterated group of additive commutators, for any positive integer $n$. These fall under a wider class of problems concerning the question of whether a noncentral subnormal subgroup of $D^{*}$ cannot be "too small", and questions about the images of (non-commutative) polynomials evaluated on central simple algebras. In the case $n=1$, both results have been proved Chebotar et al in [6. Theorem 3, theorem 6], and recently again by the authors and S. Akbari in [1, Theorem 6, Theorem 7]. Both [1, 6] and the current paper use rational polynomial identities in proving the aforementioned results. The idea is simple and clever: The key is a certain (non-commutative) polynomial $g_{n}\left(x, y_{1}, \ldots, y_{n}\right)$ that vanishes

E-mail Addresses: maghabali@gmail.com, mhbien@hcmus.edu.vn

The first author's current institute: School of Mathematics, Statistics and Computer Science, University of Tehran, Tehran, Iran 
whenever an algebraic element of degree $\leq n$ is substituted into $x$. One takes $n<\operatorname{deg} D$, substitutes a relevant rational expression into $x$ and proves that the resulting expression cannot vanish on $D \otimes_{F} L$, where $L$ is some splitting field of $D$. In [1] and [6], the expressions substituted into $x$ are single additive, resp. multiplicative, commutators on two variables, whereas here, iterated commutators are considered.

\section{Rational identities}

Let $F$ be a field and $X=\left\{x_{1}, \ldots, x_{m}\right\}$ be $m$ noncommuting indeterminates. Denote by $F\langle X\rangle$ and $F(X)$ respectively the free algebra in $X$ over $F$ and the universal division ring of fractions of $F\langle X\rangle$. A rational expression over $F$ is an element of $F(X)$. Let $R$ be an $F$-algebra. A rational expression $f$ over $F$ is said to be a rational identity of $R$ if it vanishes on all permissible substitutions from $R$. In this case, we say that $R$ satisfies the rational identity $f=0$.

Example 1.

1. It is not hard to see that (Hua's identity) $\left(x^{-1}+\left(y^{-1}-x^{-1}\right)^{-1}\right)^{-1}-x+x y x=0$ is a rational identity of every algebra over an arbitrary field $F$.

2. One can easily verify that $(x+y)^{-1}-y^{-1}\left(x^{-1}+y^{-1}\right)^{-1} x^{-1}=0$ is a rational identity of every algebra over an arbitrary field $F$.

3. It is easy to check that $\left(\left(x,(y, z) x(y, x)^{-1}\right)^{3}, z\right)=0$ vanishes on permissible substitutions of $M_{3}(F)$ for any field $F$.

A rational identity $f$ of an algebra $R$ is called nontrivial if $f$ is non-zero in $F(X)[6]$. In the special case when $R=D$ is a division ring, we have some further information: assume that $f=0$ is a rational identity of $D$. Then $f$ is non-trivial if and only if there exists a division ring $L$ containing all coefficients of $f$ and $f$ is not a rational identity of $L$. One direction of the statement is trivial, to see the other direction assume that $f$ is nontrivial. Then, it is well known that there exists a division ring $L$ with infinite center which contains $F$, and $L$ is infinite dimensional over its center. Hence by [7], $f=0$ is not a rational identity of $D$. In the example, it is easily seen that (1) and (2) are trivial, however one can verify that (3) is nontrivial.

In this paper, our algebras $R$ are central simple algebras over a field $F$. That is, $R \cong M_{n}(D)$ where $D$ is a division ring which is finite dimensional over $F$. We denote by $\mathcal{I}(R)$ the set of all nontrivial rational identities of the algebra $R$. It is known that a division $\operatorname{ring} D$ with infinite center $F$ is a finite dimensional vector space over its center if and only if $\mathcal{I}(D) \neq \emptyset[7$. Therefore, there are rings $R$ with $\mathcal{I}(R)=\emptyset$. Moreover,

Theorem 2. [3, Theorem 11] Let $F$ be an infinite field and $R$ be a central simple F-algebra with $\operatorname{dim}_{F} R=n^{2}$. Assume that $L$ is a field extension of $F$. Then $\mathcal{I}(R)=\mathcal{I}\left(M_{n}(F)\right)=\mathcal{I}\left(M_{n}(L)\right)$.

We consider the following example of a rational expression which is important in this paper. Given a positive integer $n$ and $n+1$ noncommutative indeterminates $x, y_{1}, \ldots, y_{n}$, put

$$
g_{n}\left(x, y_{1}, \ldots, y_{n}\right)=\sum_{\delta \in S_{n+1}} \operatorname{sign}(\delta) x^{\delta(0)} y_{1} x^{\delta(1)} y_{2} x^{\delta(2)} \ldots y_{n} x^{\delta(n)}
$$

where $S_{n+1}$ is the symmetric group of $\{0, \ldots, n\}$ and $\operatorname{sign}(\delta)$ is the sign of permutation $\delta$. This is a rational expression defined in [5] as a mean to test whether an element is algebraic of degree $n$. This rational expression may be considered as a generalisation of the characteristic polynomials of matrices of degree $n$ over a field.

Lemma 3. Let $R=M_{n}(D)$ be a central simple algebra over its center $F$. For any element a $\in$, the following conditions are equivalent.

1. The element a is algebraic over $F$ of degree less than or equal to $n$.

2. $g_{n}\left(a, r_{1}, r_{2}, \ldots, r_{n}\right)=0$ for any $r_{1}, r_{2}, \ldots, r_{n} \in R$.

Proof. It is just a corollary of [5, Corollary 2.3.8].

\section{Subfields generated by the elements in the $n$-th multiplicative derived subgroup}

Let $n$ be a positive integer and let $x_{1}, \ldots, x_{2^{n}}$ be $2^{n}$ indeterminates. We will define a special rational polynomial $u_{n}\left(x_{1}, \ldots, x_{2^{n}}\right)$ successively as follows: set $u_{1}\left(x_{1}, x_{2}\right)=\left(x_{1}, x_{2}\right)=x_{1} x_{2} x_{1}^{-1} x_{2}^{-1}$ and assume that $u_{n-1}\left(x_{1}, \ldots, x_{2^{n-1}}\right)$ is defined. Then we put

$$
u_{n}\left(x_{1}, \ldots, x_{2^{n}}\right)=u_{1}\left(u_{n-1}\left(x_{1}, \ldots, x_{2^{n-1}}\right), u_{n-1}\left(x_{2^{n-1}+1}, \ldots, x_{2^{n}}\right)\right) .
$$

This polynomial relates to the solvability of a group: if $G$ is a solvable group of length $\leq n$, that is $G^{(n)}=1$, then $u_{n}\left(a_{1}, \ldots, a_{2^{n}}\right)=1$ for every $a_{1}, \ldots, a_{2^{n}} \in G$. In fact, we show the following result. 
Lemma 4. Let $u_{n}$ be as above. If $G$ is a group with (multiplicative) derived series

$$
G \supseteq G^{\prime} \supseteq \cdots \supseteq G^{(n)} \supseteq \ldots,
$$

then $u_{n}\left(a_{1}, \ldots, a_{2^{n}}\right) \in G^{(n)}$ for $a_{1}, \ldots, a_{2^{n}} \in G$.

Proof. We prove the lemma by induction on $n$. Assume that $G$ is a group and $a_{1}, a_{2} \in G$. One has $a_{1} a_{2} a_{1}^{-1} a_{2}^{-1} \in G^{\prime}$, which implies that $u_{1}\left(a_{1}, a_{2}\right) \in G^{\prime}$. Hence, the lemma holds for $u_{1}$ and for the group $G$. Assume that for every group $H, u_{n-1}\left(a_{1}, \ldots, a_{2^{n-1}}\right) \in H^{(n-1)}$ for every $a_{1}, \ldots, a_{2^{n-1}} \in H$. We must prove that for every group $G, u_{n}\left(b_{1}, \ldots, b_{2^{n}}\right) \in G^{(n)}$ for every $b_{1}, \ldots, b_{2^{n}} \in G$. This follows immediately from the definitions of $u_{n}$ and $\left.G^{(} n\right)$ by induction on $n$.

Lemma 5. Let $K$ be an infinite field and $m>1$. For any positive integer $n$ and every non-scalar matrix $C \in S L_{m}(K)$, there exist non-scalar matrices $A_{1}, \ldots, A_{2^{n}} \in S L_{m}(K)$ such that $C=u_{n}\left(A_{1}, \ldots, A_{2^{n}}\right)$.

Proof. We show the lemma by induction on $n$. Assume that $n=1$. It is well-known that every non-scalar matrix in $S L_{m}(K)$ is a single commutator 14. Hence there exist non-scalar matrices $A_{1}, A_{2} \in S L_{m}(K)$ such that $C=A_{1} A_{2} A_{1}^{-1} A_{2}^{-1}$. Thus, the statement holds in case $n=1$. Assume that the statement is true for $n-1$, that is, for every non-scalar matrix $C \in S L_{m}(K)$ there exist non-scalar matrices $A_{1}, \ldots, A_{2^{n-1}} \in S L_{m}(K)$ such that $C=u_{n-1}\left(A_{1}, \ldots, A_{2^{n-1}}\right)$. Now by the induction hypothesis for every non-scalar matrix $C$, there exist non-scalar matrices $B_{1}, B_{2}, A_{1}, \ldots, A_{2^{n}} \in S L_{m}(K)$ such that $C=u_{1}\left(B_{1}, B_{2}\right), B_{1}=u_{n-1}\left(A_{1}, \ldots, A_{2^{n-1}}\right)$, and $B_{2}=u_{n-1}\left(A_{2^{n-1}+1}, \ldots, A_{2^{n}}\right)$. Therefore,

$$
\begin{aligned}
C & =u_{1}\left(B_{1}, B_{2}\right) \\
& =u_{1}\left(u_{n-1}\left(A_{1}, \ldots, A_{2^{n-1}}\right), u_{n-1}\left(A_{2^{n-1}+1}, \ldots, A_{2^{n}}\right)\right) \\
& =u_{n}\left(A_{1}, \ldots, A_{2^{n}}\right) .
\end{aligned}
$$

This implies that the statement is true in case $n$.

Before showing the main result of this section, we recall the following well-known lemma.

Lemma 6. [10, Page 242] Let $D$ be a division ring with center $F$ and $K$ be a subfield of $D$ containing $F$. If $\operatorname{dim}_{F} D=m^{2}$, then $\operatorname{dim}_{F} K \leq m$. The equality holds if and only if $K$ is a maximal subfield of $D$.

Theorem 7. Let $D$ be a division ring finite dimensional over a its center F. For any positive integer $n$ there exists $a \in D^{(n)}$, the $n$-th multiplicative derived subgroup, such that $F(a)$ is a maximal subfield of $D$.

Proof. If $F$ is finite, then $D$ is also finite and we have nothing to prove. Suppose that $F$ is infinite and $\operatorname{dim}_{F} D=m^{2}$. By Lemma 6, it suffices to show that there exists $a \in D^{(n)}$ such that $\operatorname{dim}_{F} F(a) \geq m$. Indeed, put

$$
\ell=\max \left\{\operatorname{dim}_{F} F\left(u_{n}\left(a_{1}, \ldots, a_{2^{n}}\right)\right) \mid a_{1}, \ldots, a_{2^{n}} \in D^{*}\right\} .
$$

Applying Lemma 3 we see that $g_{\ell}\left(u_{n}\left(a_{1}, \ldots, a_{2^{n}}\right), r_{1}, \ldots, r_{\ell}\right)=0$, for any $r_{1}, \ldots, r_{\ell} \in D$ and $a_{1}, \ldots, a_{2^{n}} \in D^{*}$. In other words,

$$
g_{\ell}\left(u_{n}\left(x_{1}, \ldots, x_{2^{n}}\right), y_{1}, \ldots, y_{\ell}\right)=0
$$

is a rational identity of $D$. It is not hard to verify that $g_{\ell}\left(u_{n}\left(x_{1}, \ldots, x_{2^{n}}\right), y_{1}, \ldots, y_{\ell}\right)$ is a non-zero element of $F\left(x_{1}, \ldots, x_{2^{n}}, y_{1}, \ldots, y_{\ell}\right)$, (see [9], Theorem 3.4]). Hence, by Theorem 2] it is also a rational identity of $M_{n}(F)$. This yields that $g_{\ell}\left(u_{n}\left(A_{1}, \ldots, A_{2^{n}}\right), B_{1}, \ldots, B_{\ell}\right)=0$, for all matrices $A_{i} \in G L_{m}(F)$ and $B_{i} \in M_{m}(F)$. In the view of Lemma 3, $u_{n}\left(A_{1}, \ldots, A_{2^{n}}\right)$ is algebraic over $F$ of degree $\leq \ell$ for every $A_{1}, \ldots, A_{2^{n}} \in M_{m}(F)$. Now consider the $m \times m$-matrix $T=\left(t_{i j}\right)_{1 \leq i, j \leq m}$ as follows: if $j=i$ or $j=i+1$, then $t_{i j}=1$; otherwise $t_{i j}=0$. It is easy to see that $T \in S L_{m}(F)$ and $T$ is algebraic of degree $m$ over $F$. By Lemma 5 , one can find matrices $A_{1}, \ldots, A_{2^{n}} \in S L_{m}(F)$ such that $u_{n}\left(A_{1}, \ldots, A_{2^{n}}\right)=T$. Hence, $\ell \geq m$. This completes the proof.

\section{Subfields generated by the elements in the $n$-th additive derived subgroup}

Let $n$ be a positive integer and let $x_{1}, \ldots, x_{2^{n}}$ be $2^{n}$ indeterminates. We define a polynomial $v_{n}\left(x_{1}, \ldots, x_{2^{n}}\right)$ successively as follows: set $v_{1}\left(x_{1}, x_{2}\right)=\left[x_{1}, x_{2}\right]=x_{1} x_{2}-x_{2} x_{1}$. Assume that $v_{n-1}\left(x_{1}, \ldots, x_{2^{n-1}}\right)$ is defined. Then we put

$$
v_{n}\left(x_{1}, \ldots, x_{2^{n}}\right)=v_{1}\left(v_{n-1}\left(x_{1}, \ldots, x_{2^{n-1}}\right), v_{n-1}\left(x_{2^{n-1}+1}, \ldots, x_{2^{n}}\right)\right) .
$$


Lemma 8. Let $R$ be an algebra with additive derived series

$$
R \supseteq R_{1} \supseteq \cdots \supseteq R_{n} \supseteq \ldots
$$

If $v_{n}$ is defined as above, then $v_{n}\left(a_{1}, \ldots, a_{2^{n}}\right) \in R_{n}$ for $a_{1}, \ldots, a_{2^{n}} \in R$.

Proof. The proof is similar to that of Lemma 4

Lemma 9. Let $K$ be a field and $m>1$ such that char $K \nmid m$. For any positive integer $n$, and every matrix $C \in$ $M_{m}(K)$ with zero-trace, there exist matrices $A_{1}, \ldots, A_{2^{n}} \in M_{m}(K)$ whose trace is zero and $C=v_{n}\left(A_{1}, \ldots, A_{2^{n}}\right)$.

Proof. The idea of the proof is similar to that of Lemma 5. We prove the lemma by induction on $n$. Assume that $n=1$. In the view of [2], which states that every matrix in $M_{n}(K)$ with zero-trace is a single additive commutator, there exist $A_{1}, A_{2} \in M_{m}(K)$ such that $C=A_{1} A_{2}-A_{2} A_{1}$. Set $B_{1}=A_{1}-\frac{\operatorname{tr}\left(A_{1}\right)}{m} I_{m}$ and $B_{2}=$ $A_{2}-\frac{\operatorname{tr}\left(A_{2}\right)}{m} I_{m}$, where by $\operatorname{tr}(A)$ we mean the trace of $A$. We have $C=B_{1} B_{2}-B_{2} B_{1}=v_{1}\left(B_{1}, B_{2}\right)$ and $\operatorname{tr}\left(B_{1}\right)=\operatorname{tr}\left(B_{2}\right)=0$. Hence, the statement holds in case $n=1$. The general case follows by induction on $n$, similarly to the proof of Lemma 5 .

The following result is the goal of this section. Note that the same result as Theorem 7 , however, follows from the case $n=1$, proved in [1] and [6], since by a theorem of Amitsur and Rowen $D_{1}=D_{2}=D_{3}=\ldots$. 4]. However, the proof yields the slightly stronger claim that a single depth- $n$ iterated additive commutator would generate a maximal subfield, which does not follow from $D_{1}=D_{2}=D_{3}=\ldots$

Theorem 10. Let $D$ be a division ring finite dimensional over its center $F$ of characteristic either zero or a prime $p$ such that $p \nmid \operatorname{dim}_{F} D$. For any positive integer $n$, there exists a depth-n iterated additive commutator which generates a maximal subfield of $D$.

Proof. First note that if $\operatorname{char} F=0$, then by a result due to Amitsur and Rowen [4] we have $D_{1}=D_{2}=\cdots$. Hence, in this case the result follows from [1, Theorem 7]. In the case of $\operatorname{char} F=p>0$, the proof is similar to the one of Theorem 7 . We assume that $F$ is infinite since if $F$ is finite, then $D$ is also finite and there is nothing to prove. Suppose $\operatorname{dim}_{F} D=m^{2}$. In the view of Lemma 6 , it suffices to show that there exists $a \in D_{n}$ such that $\operatorname{dim}_{F} F(a) \geq m$. Indeed, put

$$
\ell=\max \left\{\operatorname{dim}_{F} F\left(v_{n}\left(a_{1}, \ldots, a_{2^{n}}\right)\right) \mid a_{1}, \ldots, a_{2^{n}} \in D^{*}\right\} .
$$

According to Lemma 3 we see that $g_{\ell}\left(v_{n}\left(a_{1}, \ldots, a_{2^{n}}\right), r_{1}, \ldots, r_{\ell}\right)=0$, for any $r_{1}, \ldots, r_{\ell} \in D$ and $a_{1}, \ldots, a_{2^{n}} \in$ $D^{*}$. In other words,

$$
g_{\ell}\left(v_{n}\left(x_{1}, \ldots, x_{2^{n}}\right), y_{1}, \ldots, y_{\ell}\right)=0
$$

is a polynomial identity of $D$, so it is also a rational identity of $M_{n}(F)$ (Lemma 2). Note that it is easily seen that $g_{\ell}\left(v_{n}\left(x_{1}, \ldots, x_{2^{n}}\right), y_{1}, \ldots, y_{\ell}\right)$ is a non-zero element of $F\left(x_{1}, \ldots, x_{2^{n}}, y_{1}, \ldots, y_{\ell}\right)$ (see 9 , Theorem 3.4]). This yields that

$$
g_{\ell}\left(v_{n}\left(A_{1}, \ldots, A_{2^{n}}\right), B_{1}, \ldots, B_{\ell}\right)=0,
$$

for all matrices $A_{i}, B_{i} \in M_{m}(F)$. According to Lemma 3, $v_{n}\left(A_{1}, \ldots, A_{2^{n}}\right)$ is algebraic over $F$ of degree $\leq \ell$ for every $A_{1}, \ldots, A_{2^{n}} \in M_{m}(F)$. Now consider the $m \times m$-matrix $T=\left(t_{i j}\right)_{1 \leq i, j \leq m}$ defined by $t_{i(i+1)}=1$ and $t_{i j}=0$ if $j \neq i+1$. We can show that $\operatorname{tr}(T)=0$ and $T$ is algebraic of degree $m$ over $F$. By Lemma 9, there exist matrices $A_{1}, \ldots, A_{2^{n}} \in M_{m}(F)$ such that $v_{n}\left(A_{1}, \ldots, A_{2^{n}}\right)=T$. Hence, $\ell \geq m$ and this completes the proof.

Acknowledgment: The research of the first author was supported with ERC [grant number 320974]. The second author was funded by Vietnam National University HoChiMinh City (VNU-HCM) under grant no. C2018-18-03.

\section{References}

[1] M. Aaghabali, S. Akbari and M.H. Bien, Divison algebras with left algebraic commutators, Algebr. Represent. Theor., 21 (4) (2018) 807-816.

[2] A.A. Albert and B. Muckenhoupt, On mtrices of trace zeros, Michigan Math. J., 4 (1) (1957) 1-3.

[3] S.A. Amitsur, Rational identities and applications to algebra and geometry, J. Algebra, 3 (1966) $304-359$.

[4] S.A. Amitsur, L.H. Rowen, Elements of Reduced Trace 0, Israel J. Math., 87 (1994) 161-179.

[5] K.I. Beidar, W.S. Martindale and A.V. Mikhalev, Rings with Generalized Identities, Marcel Dekker, Inc., New York-Basel-Hong Kong, 1996. 
[6] M.A. Chebotar, Y. Fong and P.H. Lee, On division rings with algebraic commutators of bounded degree, Manuscripta Math., 113 (2004) 153-164.

[7] K. Chiba, Generalized rational identities of subnormal subgroups of skew fields, Proc. Amer. Math. Soc., 124 (6) (1996) 1649-1653.

[8] P.M. Cohn, Free rings and their relations, Academic Press, New York and London, 1971.

[9] B.X. Hai, T.H. Dung and M.H. Bien, Almost subnormal subgroups in division rings with generalized algebraic rational identities, submitted. (https://arxiv.org/pdf/1709.04774.pdf)

[10] T.Y. Lam, A First Course in Noncommutative Rings, 2nd Ed, GTM, No. 131, Springer-Verlage, New York, 2001.

[11] M. Mahdavi-Hezavehi, Commutators in division rings revisited, Bull. Iranian Math. Soc., 26 (2) (2000) 7-88.

[12] M. Mahdavi-Hezavehi, Extension of valuations on derived groups of division rings, Comm. Algebra, 23 (3) (1995) 927-940.

[13] M. Mahdavi-Hezavehi, S. Akbari-Feyzaabaadi, M. Mehraabaadi and H. Hajie-Abolhassan, Commutators in division rings II, Comm. Algebra, 23 (8) (1995) 2881-2887.

[14] R.C. Thompson, Commutators in the special and general linear groups, Trans. Amer. Math. Soc., 101 (1) (1961) 16-33. 